\title{
Qualidade de Vida e Aspectos Socioeconômicos em Diabéticos Tipo 1
}

\section{original}

\author{
Angelo Fontes Araújo \\ Maria Eliane Alves Souza \\ Carlos Alberto Menezes
}

Curso de Medicina do Departamento de Ciências da Saúde da Universidade Estadual de Santa Cruz (UESC), Ilhéus, BA,

Brasil

\section{RESUMO}

Objetivos: Avaliar a qualidade de vida (OV) em diabéticos tipo 1 assistidos na Associação dos Diabéticos de Itabuna, BA (ASDITA), e verificar suas relações com as variáveis clínicas e sociodemográficas (escolaridade, renda familiar e ocupação). Métodos: Foram estudados 34 pacientes $(\gamma=88 \%, \varepsilon=$ $5 \% ; 53 \%$ mulheres, média de idade $20,4 \pm 8,4$ anos e média de tempo de diagnóstico 5,7 $\pm 4,9$ anos), por meio de entrevista e antropometria (medidas de peso e altura). Resultados: O perfil sociodemográfico da amostra revelou renda familiar per capita de $0,70 \pm 0,56$ salários mínimos, $53 \%$ possuíam o ensino fundamental, $64,7 \%$ eram estudantes. Em média, a qualidade de vida (OV) foi regular (58,8\%). Não interferiram na QV: escolaridade, ocupação, etnia, índice de massa corpórea, número de aplicações diárias de insulina e perfil de monitoração da glicemia capilar. Associaram-se a pior QV: sexo feminino, ser adulto, tempo de diagnóstico maior ou igual a dez anos e baixa renda familiar. Conclusões: A OV destes pacientes foi inferior ao descrito na literatura, entretanto, estudos adicionais são necessários para comparações com diabéticos em condições socioeconômicas e culturais semelhantes às observadas nesta pesquisa. (Arq Bras Endocrinol Metab 2008; 52/7:1124-1130)

Descritores: Diabetes melito tipo 1; Qualidade de vida; Fatores socioeconômicos; Psicologia

\section{ABSTRACT}

Quality of Life and Socio-economical Aspects of Diabetics Type 1.

Objectives: This research has appraised the quality of life (OL) of diabetics type 1 member of Associação dos Diabéticos de Itabuna (ASDITA) and has verified their correlation between clinic variable and social-demographic situation of this group. Research Design and Methods: Thirty-four patients $(\gamma=88 \%, \varepsilon=5 \%$; $53 \%$ female, age $20.4 \pm 8.4$ years, diagnostic time $5.7 \pm 4.9$ year) have participated in this research, data showed weight and height after the interview and measuring. Results: The social-demographic profiles of this group revealed family income of $0.7 \pm 0.56$ of minimal salary, $53 \%$ had elementary school level and $64.7 \%$ were students. In avarage, the $\mathrm{OL}$ was regular $(58.8 \%)$. OL has not been influenced by the level of education, ethnology, occupation, BMI, daily shot of insulin, or glicemic monitorization profile but woman, adult, diagnostic time more than ten years, little family income have had the worst QL. Conclusions: The OL of the searched patients has been less than what several publications have showed, but additional researches are necessary to have better comparisons between diabetics with socio-economical and cultural conditions similar to the ones observed in this research. (Arq Bras Endocrinol Metab 2008; 52/7:1124-1130)

Recebido em 27/6/2007 Aceito em $7 / 7 / 2008$
Keywords: Type 1 diabetes mellitus; Quality of life; Socio-economics factors; Psychology 


\section{INTRODUÇÃO}

S egundo a Organização Mundial da Saúde (OMS), a qualidade de vida (QV) é “a percepção do indivíduo de sua posição na vida, no contexto da cultura e sistema de valores nos quais ele vive e em relação aos seus objetivos, expectativas, padrões e preocupações" (1). Contudo, a definição de QV não é consenso na literatura. É reconhecida sua construção multidimensional, incluindo aspectos psicoemocionais, socioeconômicos, culturais, políticos, ambientais e da saúde. Outro aspecto importante é a subjetividade e, portanto, a individualidade, na sua avaliação (2-4).

$\mathrm{O}$ diabetes pode afetar adversamente o funcionamento psicossocial e a QV dos indivíduos acometidos, repercutindo nos domínios físico, social e psicoemocional (2-8). Esse impacto dependerá da percepção do paciente e da sua família, da forma como lidam com o autocuidado e com o manejo da doença e do funcionamento familiar como um todo. Os amigos são importante recurso de apoio social aos diabéticos, principalmente entre os adolescentes $(5,6)$.

Os prejuízos no funcionamento físico incluem as complicações a curto e longo prazos, os sintomas físicos, as mudanças no estilo de vida pelas demandas do tratamento e os efeitos colaterais das medicações (3). O quadro psicoemocional pode ser composto por preocupação, frustração e desesperança com o caráter crônico da doença e suas complicações; sobrecarga, esgotamento ou desânimo com seu manejo. Citam-se, ainda, baixa auto-estima, inferioridade, ansiedade e depressão $(3,4,6)$. Entre os aspectos sociais estão o custo financeiro da doença, a sensação do paciente acerca do grau de apoio social que recebe e da qualidade e nível de conflito das relações interpessoais e familiares (3-5).

O diabetes melito tipo 1 ( $\mathrm{DMl})$ é uma doença crônica com início geralmente na infância e na adolescência, podendo prejudicar o crescimento, o desenvolvimento e o ajuste psicossocial de seus portadores (5-8). A impulsividade das crianças e dos adolescentes confronta-se com a racionalidade exigida pelo manejo da doença, tornando as condições emocionais mais difíceis para este grupo (6). No processo de ajuste, a falta de informação e a superproteção são prejudiciais, enquanto a transferência gradual de responsabilidades de autocuidado é facilitadora, permitindo alcançar a auto-suficiência (5-7).

Alguns autores sugerem que a percepção da QV deva ser mensurada na prática clínica, viabilizando o suporte com enfoque em parâmetros biomédicos e psicossociais
(2-3). O objetivo deste trabalho foi avaliar a QV dos pacientes com DMl assistidos na Associação dos Diabéticos de Itabuna (ASDITA), BA, centro de referência na região sul da Bahia, e verificar suas relações com as variáveis clínicas e sociodemográficas desta população.

\section{MÉTODOS}

Trata-se de uma pesquisa exploratória e transversal com $\mathrm{n}=34$ pacientes com DMl $(\gamma=88 \%, \varepsilon=5 \%)$ cadastrados na ASDITA. Os pacientes foram devidamente informados sobre a pesquisa e assinaram termo de consentimento. O protocolo do estudo foi aprovado pela Comissão de Ética da Universidade Estadual de Santa Cruz (UESC). O critério de inclusão foi ser paciente com DMl cadastrado na ASDITA, capaz de responder sozinho à entrevista aplicada. Os participantes foram selecionados aleatoriamente durante as reuniões educativas mensais promovidas nesta instituição.

Os pacientes foram classificados conforme a idade, o índice de massa corpórea (IMC) e a cor/etnia. Quanto à idade tem-se: crianças (< 10 anos), adolescentes (10-19 anos) e adultos (> 19 anos) (9). Para o IMC, os adultos foram classificados em: normais $\left(18,5 \mathrm{~kg} / \mathrm{m}^{2} \leq\right.$ IMC $\left.\leq 24,9 \mathrm{~kg} / \mathrm{m}^{2}\right)$, sobrepeso $\left(25 \mathrm{~kg} / \mathrm{m}^{2} \leq\right.$ IMC $\leq$ $\left.29,9 \mathrm{~kg} / \mathrm{m}^{2}\right)$ e obesos (IMC $\left.\geq 30 \mathrm{~kg} / \mathrm{m}^{2}\right)(10)$. Para as crianças e os adolescentes foram adotadas as recomendações do Centers for Disease Control and Prevention (CDC) e do National Center for Health Statistics (NCHS), segundo os quais, após o cálculo do IMC, projeta-se o valor obtido para cada indivíduo em gráfico contendo curvas de percentis de IMC. Há um gráfico específico para cada sexo, no qual, no eixo das abscissas, tem-se a variável idade. Dessa forma, entre as crianças e os adolescentes, o IMC é ajustado para idade e sexo. Os valores de referência são: normal (percentil 5 $\leq$ IMC < percentil 85), risco de sobrepeso (percentil 85 $\leq$ IMC $<$ percentil 95) e sobrepeso (IMC $\geq$ percentil $95)(11,12)$. Os participantes foram classificados quanto a cor/etnia, conforme Maio e cols. (13).

Os participantes da pesquisa auto-avaliaram sua QV por meio de entrevista estruturada, intermediada por estudante de medicina previamente treinado. Em seguida, foram submetidos a antropometria (medidas do peso e da altura). Estas etapas da pesquisa foram realizadas individualmente, nos consultórios da ASDITA. A entrevista foi composta por 36 questões nos domínios geral, físico, social, emocional e psicológico. As questões que compuseram a entrevista foram extraídas de 
questionários usados em estudos de outros autores e adaptadas para a realidade local (14-17).

A entrevista passou por validação interna pelo método de Cronbach (alpha $=0,85$ ) e por validação consultiva por meio de estudo-piloto com funcionários e estudantes da UESC portadores de diabetes. Este tipo de validação tem por finalidade a estruturação coesa e coerente das questões do instrumento, a fim de obter informações precisas, completas e relevantes dos dados coletados $(18,19)$.

Por meio da análise da entrevista estruturada obteve-se o índice para mensurar a QV. O índice é a quantificação da QV descrita pelo paciente, uma vez que este aponta, em cada quesito dos domínios, os pesos que correspondem ao seu estado. O objetivo é representar dados multivariados, sujeitos a importante e complexa relação, propiciando caminho pelo qual o pesquisador possa, rápida e sistematicamente, obter informações sobre o estado de QV (20).

$\mathrm{Na}$ elaboração do índice foram definidos cinco domínios de percepção dos pacientes (geral, físico, social, emocional e psicológico), que compuseram o quadro de avaliação da QV. Cada domínio foi composto por um conjunto específico de variáveis presentes nos quesitos da entrevista. Para a construção do índice foi calculado o escore de cada um dos quesitos, os quais possuíam opções de respostas organizadas na forma de escalas ordinais: ótimo, bom, regular, ruim e péssimo. Estas escalas foram transformadas em intervalares, atribuindo-se à escala ordinal os valores 5,4 , 3,2 e 1 . O valor 5 foi atribuído à resposta mais positiva e 1 à mais negativa. Os pesos diferenciados $\left(\mathrm{w}_{\mathrm{i}}\right) \mathrm{de}$ cada variável no índice foram obtidos por meio da explicação do fator Varimax. Os pesos foram construídos por meio do recurso Algorithmic Scaling (ALSCAL), do programa estatístico Statistical Package the Social Science (SPSS).

O índice de QV foi categorizado em bom, regular ou ruim, com base na distribuição das respostas em torno da média e seu desvio obtidos nos escores das escalas itemizadas do questionário. Por meio do pressuposto da distribuição normal, os valores de intervalo em torno da média são definidos como o aspecto mediano, e os intervalos abaixo e acima deste são considerados como os extremos, daí resultando a categorização em bom, regular e ruim $(20,21)$.

As variáveis estudadas compreenderam o índice geral de QV e o perfil clínico dos pacientes: tempo de diagnóstico, número de injeções diárias de insulina, padrão de monitoração da glicemia capilar e IMC. Para avaliação sociodemográfica foram pesquisados sexo, idade, cor/etnia, escolaridade, rendas familiar e per $\mathrm{ca}^{-}$ pita e situação ocupacional.

Utilizou-se o SPSS (release 10.0.1, 1999) para a análise estatística, que fundamentou-se em medidas resumo (proporção, média, moda e desvio-padrão) e análise bivariadas e multivariadas. As análises bivariadas consistiram de cruzamentos da variável índice de QV com cada uma das variáveis dos perfis clínico e sociodemográfico, citadas anteriormente. Os cruzamentos considerados significativos (entre o índice de QV e as variáveis rendas familiar e per capita, sexo, idade e tempo de diagnóstico) foram selecionados por meio das freqüências observadas e a distribuição dos percentuais de destaque está descrita nos resultados. A análise multivariada fatorial implementada do tipo exploratória, sem definição a priori do número de fatores, pela rotação Varimax, foi utilizada na avaliação quantitativa da $\mathrm{QV}$ por meio do índice de $\mathrm{QV}$.

\section{RESULTADOS}

\section{Caracterização da amostra}

A caracterização sociodemográfica da amostra é representada na Tabela 1 . Em relação à escolaridade, 53\% possuíam o ensino fundamental (completo ou incompleto) e quanto à ocupação, $64,7 \%$ eram estudantes.

Tabela 1. Distribuição das características sociodemográficas entre os pacientes com diabetes melito tipo 1 - Itabuna, BA 2006.

\begin{tabular}{|c|c|c|}
\hline Características & $\mathrm{n}$ & $\begin{array}{l}\text { Percentual ou média } \\
\pm \text { desvio-padrão }\end{array}$ \\
\hline \multicolumn{3}{|l|}{ Sexo } \\
\hline Masculino & 16 & $47,0 \%$ \\
\hline Feminino & 18 & $53,0 \%$ \\
\hline \multicolumn{3}{|l|}{ Cor/etnia } \\
\hline Pardos & 19 & $56,0 \%$ \\
\hline Brancos & 9 & $26,4 \%$ \\
\hline Pretos & 6 & $17,6 \%$ \\
\hline \multicolumn{3}{|l|}{ Grupos etários } \\
\hline Crianças & 2 & $5,90 \%$ \\
\hline Adolescentes & 13 & $38,2 \%$ \\
\hline Adultos & 19 & $55,9 \%$ \\
\hline Idade & - & $20,4 \pm 8,4$ anos \\
\hline Renda familiar & - & $3,3 \pm 2,9 \mathrm{SM}$ \\
\hline Renda familiar per capita & - & $0,70 \pm 0,56 \mathrm{SM}$ \\
\hline
\end{tabular}

SM: salários mínimos. 
Referente ao perfil clínico dos pacientes, o tempo de diagnóstico de DMl foi $5,7 \pm 4,9$ anos. A maioria $(70,6 \%)$ aplicava insulina 2 vezes ao dia, enquanto os que faziam uso 1 e 3 vezes ao dia foram $11,8 \%$. Apenas $17,6 \%$ monitorava a glicemia capilar diariamente ( 1 a 2 vezes). Os que não monitoravam e aqueles que a realizavam não diariamente somaram $82,4 \%$. Os adultos com sobrepeso foram $10,5 \%$ ( $5,9 \%$ do total) e não houve obesos. Entre as crianças e os adolescentes, não houve risco de sobrepeso nem sobrepeso.

\section{Avaliação da qualidade de vida}

No geral, o índice de QV foi regular $(58,8 \%)$ com igual percentual dos índices bom e ruim $(20,6 \%$, cada). Não interferiram na QV: escolaridade, etnia, situação ocupacional, IMC, número de aplicações diárias de insulina e padrão de monitoração da glicemia capilar.

A baixa renda familiar repercutiu negativamente sobre a QV, com tendência a índice ruim quando inferior a dois salários mínimos $(\mathrm{SM})$ e tendência a bom, quando superior. Somente para renda per capita na família superior a um SM houve tendência a índice de QV bom (Figura 1).

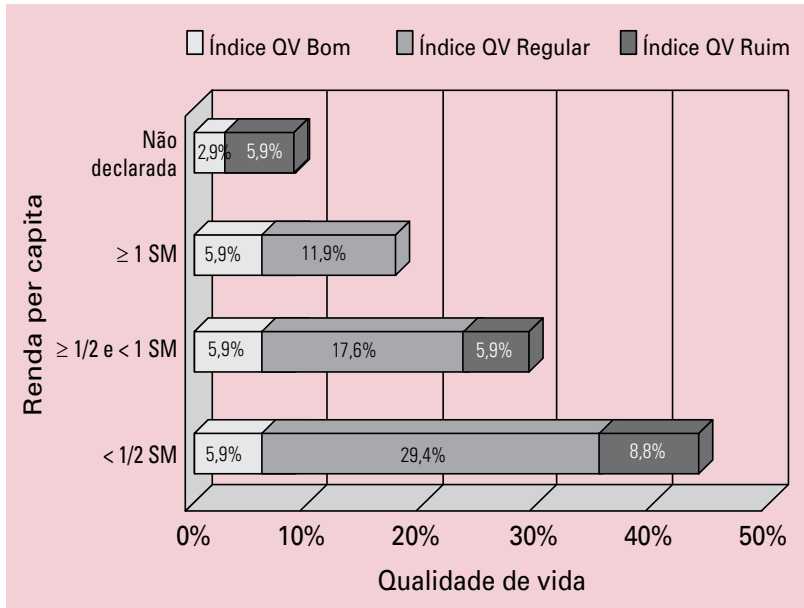

Figura 1. Índice de qualidade de vida de acordo com a renda per capita da família dos pacientes com diabetes melito tipo 1 - Itabuna, BA, 2006.

A Figura 2 ilustra o índice da QV de acordo com sexo e grupos etários. As mulheres obtiveram pior avaliação: $83,4 \%$ de índice ruim ou regular contra $75 \%$ dos homens. Os adolescentes obtiveram melhor QV que crianças e adultos. Houve ampla variação da QV por faixas etárias. O tempo de diagnóstico superior a dez anos resultou pior $\mathrm{QV}$, sem representação do índice bom (Figura 3).

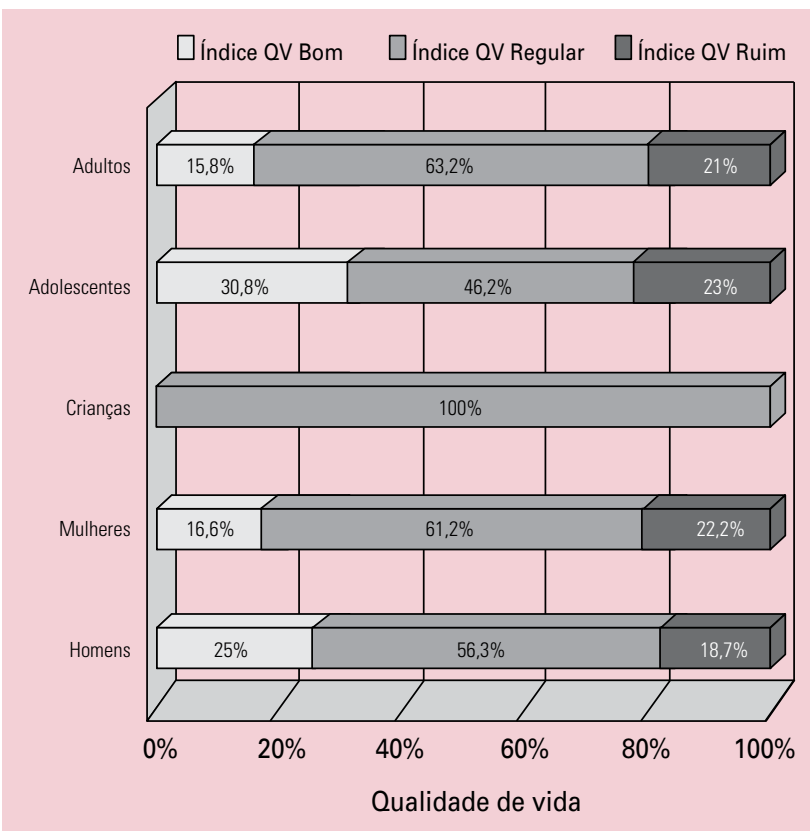

Figura 2. Índice de qualidade de vida de acordo com os grupos etários e o sexo dos pacientes com diabetes melito tipo 1 - Itabuna, BA, 2006.

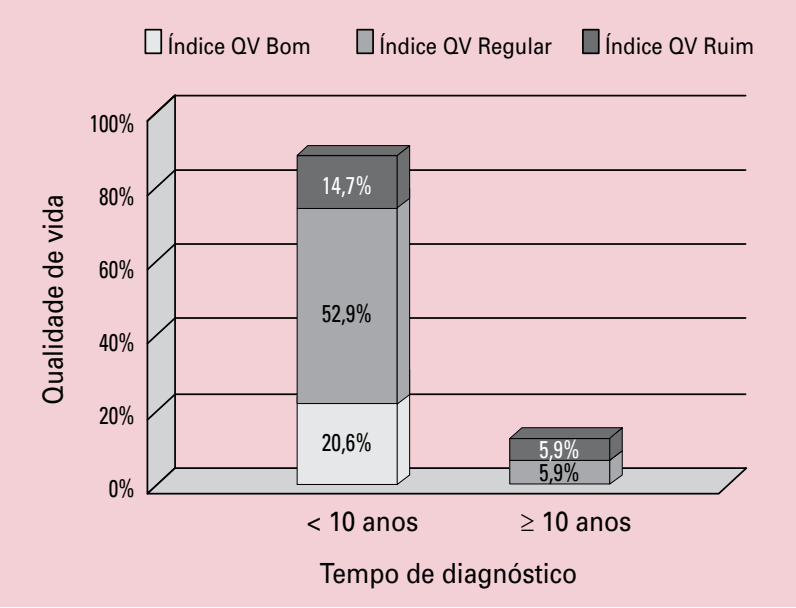

Figura 3. Índice de qualidade de vida segundo tempo de diagnóstico de diabetes melito tipo 1 - Itabuna, BA, 2006.

\section{DISCUSSÃO}

No geral, o índice de QV dos pacientes com DMl da ASDITA foi regular. Outras pesquisas encontraram melhores resultados. Em um estudo prospectivo com média de acompanhamento de 10,2 anos, $87 \%$ dos pa- 
cientes consideraram a QV como boa ou excelente (22). Em outro, com adolescentes, houve percepção positiva indicando boa QV (23). Em Minas Gerais, $60,4 \%$ dos DMl relataram lidar bem ou muito bem com sua doença, podendo sugerir que o impacto na QV destes indivíduos não foi tão significativo quanto o encontrado na amostra deste estudo (24). Entretanto, estas disparidades podem decorrer de diferenças socioeconômicas e culturais, que interferem na avaliação que os indivíduos, com a mesma doença, fazem da sua QV.

$\mathrm{O}$ nível de escolaridade e a situação ocupacional não mostraram influência considerável sobre a QV. A maioria das publicações não investigou essas variáveis (23-29). A escolaridade das mães foi maior entre os pacientes que referiram melhor QV (22).

Os dados dessa pesquisa mostraram que a $\mathrm{QV}$ foi muito variável por faixas etárias. A literatura descreve resultados diversos, como maior média de idade em pacientes com menor aceitação da doença e melhor status de saúde entre os mais jovens e menor satisfação com a vida entre os adolescentes do sexo feminino entre $12 \mathrm{e}$ 16 anos $(22,24,25)$.

Considerados os grupos etários, os adolescentes foram os que obtiveram melhor auto-avaliação nesse estudo, embora pior que em outras publicações nas quais a QV foi, no geral, boa $(23,26)$. Diferenças na percepção entre os grupos etários podem ser esperadas pelas características psicológicas próprias de cada um deles. Talvez entre os adultos já exista maior consciência sobre o diabetes e suas repercussões a longo prazo, ao mesmo tempo em que há maior experiência com situações negativas relacionadas à doença. Evidentemente, as crianças não concebem o diabetes com a mesma dimensão que os adultos, possivelmente sofrendo com repercussões mais imediatas, como limitações alimentares e incômodo com o uso da insulina, entre outros. Os adolescentes provavelmente encontram-se em situação intermediária.

As mulheres tenderam a ter pior QV. Cerca de $84 \%$ obtiveram índice ruim ou regular, o que é corroborado por outros autores. A avaliação regular ou ruim foi superior a $60 \%$ no sexo feminino, em Taiwan (22). As mulheres também apresentam avaliação negativa em idade mais precoce que os homens (24). Quando consideradas escalas de QV, o impacto do DMl foi semelhante para ambos os sexos, mas a preocupação com a doença e a percepção da saúde foi maior para as adolescentes acima de 12 anos e a satisfação foi pior para aquelas entre 12 e 16 anos (25). Faulkner e cols. (27) detectaram que adolescentes do sexo feminino com DMl expressaram menor satisfação na adolescência média $(\geq 15 \mathrm{e}<17$ anos) que as encontradas no início ou no final da adolescência. Contudo, não houve diferença entre os sexos nas escalas de preocupação e impacto relacionados ao DM.

Diversos fatores nas mulheres são citados como prováveis causas de pior controle metabólico e QV: maior preocupação com a doença, e possivelmente maior risco para depressão; entrada na puberdade ( $\mathrm{e}$ transferência de tarefas de autocuidado) em idade mais precoce; fatores psicológicos e mudanças físicas na adolescência (26).

Os dados do presente estudo revelaram efeito adverso da baixa renda sobre a $\mathrm{QV}$ dos diabéticos. Maior nível socioeconômico dos pais de diabéticos está associado a melhor QV (22). Os custos com o manejo ideal do diabetes ou o uso de insulinas especiais que proporcionam maior comodidade e melhor controle glicêmico tornam estas práticas de difícil acesso para a maioria dos diabéticos deste estudo. No Brasil, há distribuição gratuita de insulina, mas não do material para automonitoração (28).

$\mathrm{Na}$ investigação do presente estudo, a etnia não se mostrou fator importante para a QV. Publicações internacionais detectaram pior avaliação da $\mathrm{QV}$ em pacientes de minorias étnicas $(8,22,23,25-27)$. Esta consideração talvez não se aplique à realidade deste estudo em virtude da miscigenação racial no Brasil, em especial no Nordeste.

As variáveis clínicas que não interferiram na QV foram: IMC, número de aplicações diárias de insulina e padrão de monitoração da glicemia capilar. Estes resultados podem decorrer da homogeneização da amostra deste estudo nestes parâmetros.

A prevalência de adultos com sobrepeso nessa pesquisa foi de $10,5 \%$ ( $5,9 \%$ do total de participantes) e não houve obesos. Entre as crianças e os adolescentes, nenhum participante foi classificado como risco de sobrepeso nem como sobrepeso pelos critérios do CDC e do NCHS. As taxas descritas por outros autores são maiores. Moraes e cols. (29) encontraram prevalência de sobrepeso e obesidade entre os adultos com DMl de, respectivamente, $21 \%$ e $2,9 \%$, e risco de sobrepeso e sobrepeso em crianças e adolescentes com DMl de 13,8\% e 3\%. Os índices de sobrepeso encontrados por Arcanjo e cols. (30) foram: em crianças $33,3 \%$, em adolescentes $4,3 \%$ e em adultos $18,6 \%$. Há relato de associação significativa e inversa entre o IMC e a escala de satisfação da 
QV (23). O IMC elevado foi relacionado a pior QV em uma pesquisa multicêntrica internacional (25).

Chama a atenção na amostra deste estudo o elevado percentual de pacientes que não realizam adequadamente a monitoração glicêmica $(82,4 \%)$. Em Londrina, PR, a monitoração, ressalte-se, considerada na glicosúria e glicemia capilar, também ficou muito aquém do ideal, com $63,4 \%$ dos pacientes realizando testes até sete vezes semanais, $20,63 \%$ de 15 a 21 , e somente um paciente $(1,6 \%)$ de 29 a 35 testes semanais (31). Silveira e cols. (32), em pesquisa com pacientes de 11 municípios do Rio Grande do Sul, encontraram prevalência de $61 \%$ de automonitoração, destes apenas $27 \%$ realizavam o teste antes das principais refeições e $73 \%$ dos casos por meio da glicemia capilar. Quanto à interferência da monitoração glicêmica na $\mathrm{QV}$, a sua realização sistemática foi associada com dificuldades de lidar com a doença nos diabéticos de Minas Gerais, enquanto a nossa pesquisa não encontrou relação entre estas variáveis (24).

Trabalhos brasileiros mostram variações no número de aplicações diárias de insulina, sendo de duas para $79,36 \%$ da amostra de Almeida e cols. (31) e uma para $47 \%$ na de Silveira e cols. (32). Nesta pesquisa, 70,6\% dos pacientes realizavam duas aplicações diárias de insulina. Em relação às aplicações e à QV, pesquisa com adolescentes corroborou os achados do presente estudo, enquanto em outra, o uso de três ou mais injeções diárias associou-se a melhor auto-avaliação da saúde, parâmetro utilizado para mensurar a QV $(22,25)$. Outra publicação não encontrou diferença significativa na QV de pacientes com tratamento em bomba de insulina daqueles com múltiplas injeções (23).

A monitoração glicêmica rigorosa e a terapia insulínica intensiva são partes do tratamento preconizado para o DMl com objetivo de estreito controle metabólico para prevenir complicações crônicas. A literatura acerca da relação entre esse controle e a QV mostra resultados divergentes, embora dois estudos bem desenhados e com amostra considerável tenham encontrado associação positiva entre as duas variáveis $(22,25)$.

No presente estudo, o tempo de diagnóstico igual ou maior que dez anos levou à tendência negativa da QV. A presença da doença por mais de cinco anos já foi descrita como fator de risco para baixa aceitação da doença (24). O menor tempo de doença foi associado à melhor $\mathrm{QV}$ em outras pesquisas, entretanto, o tempo de doença não interferiu no impacto do diabetes, quando considerado isoladamente (22-25).

\section{CONCLUSÕES}

A qualidade de vida nos pacientes com DMl pesquisados foi avaliada como regular, resultado inferior às estatísticas de outros autores. Infelizmente, os trabalhos de literatura ainda são escassos, em especial com populações de perfil socioeconômico e cultural mais próximos da nossa realidade, o que permitiria comparações mais fiéis. Dessa forma, reforça-se a necessidade de estudos deste tipo nas diferentes regiões do país. Conclui-se que os fatores associados a pior $\mathrm{QV}$ foram: sexo feminino, tempo de diabetes maior ou igual a dez anos, ser adulto e baixas rendas familiar e per capita.

Agradecimentos. Agradecemos à Dra. Marluce Leão, endocrinologista da ASDITA, pelo apoio fundamental à realização da pesquisa nesta Instituição. Às médicas Carla Cristina da Cunha Reis e Érica Maria Pinto de Queiroz, pelo auxílio na coleta dos dados. Os autores declaram não haver conflitos de interesse científico neste artigo.

\section{REFERÊNCIAS}

1. WHOQL Group. The development of the World Health Organization quality of life assessment instrument (the WHOQOL). In: Orley J, Kuyken W, editors. Quality of Life Assessment: international perspectives. Heigelberg: Springer Verlag; 1994. p. 41-60.

2. Snoek FJ. Quality of Life: a closer look at measuring patients well-being. Diabetes Spectr. 2000;13(1):24-8.

3. Polonsky WH. Aspectos emocionais e da qualidade de vida do tratamento do diabetes. Curr Diab Rep Lat Am. 2002;1:388-96.

4. Polonsky WH. Understanding and assessing diabetes-specific quality of life. Diabetes Spectr. 2000;13(1):36-41.

5. Guthrie DW, Bartsocas C, Jarosz-Chabot $P$, Konstantinova M. Psychosocial issues for children and adolescents with diabetes: overview and recommendations. Diabetes Spectr. 2003;16(1):7-12.

6. Marcelino DB, Carvalho MDB. Reflexões sobre diabetes tipo 1 e sua relação com o emocional. Psicol Reflex Crit. 2005;18(1):72-7.

7. Velarde-Jurado E, Ávila-Figueroa C. Evaluación de la calidad de vida en el adolescente con enfermidad crónica. Bol Med Hosp Infant Mex. 2001;58(6):399-408.

8. Delamater AM. Quality of life in youths whit diabetes. Diabetes Spectr. 2000;13(1):42-6.

9. Organização Panamericana de Saúde. La salud del adolescente y el joven em las Americas. Washington DC: OPS; 1985 (Publ. Cient. no 489).

10. World Health Organization. Obesity: preventing and managing the global epidemic. Geneva: WHO; 1997.

11. Centers for Disease Control and Prevention. About BM for children and teens. Disponível em: http://www.cdc. gov/nccdphp/dnpa/bmi/childrens_BMI/about_ childrens_ BMI.htm. 2006 
12. Centers for Disease Control and Prevention/National Center for Healt Statistics. NCHS Growth Charts United States. Disponivel em: http://www.cdc.gov/growthcharts, 2000.

13. Maio MC, Monteiro S, Chor D, Faerstein E, Lopes CS. Cor/ raça no estudo pró-saúde: resultados comparativos de dois métodos de autoclassificação no Rio de Janeiro, Brasil. Cad Saude Publica. 2005;21(1):171-80.

14. Fleck MPA, Fachel O, Louzada S, Xavier M, Chachamovich E, Vieira G, et al. Desenvolvimento da versão em português do instrumento de avaliação de qualidade de vida da Organização Mundial da Saúde (WHOQOL-100) 1999. Rev Bras Psiquiatr. 1999;21(1):19-28.

15. Fleck MPA, Louzada S, Xavier M, Chachamovich E, Vieira G, Santos $L$, et al. Aplicação da versão em português do instrumento de avaliação de qualidade de vida da organização mundial da saúde (WHOQOL-100). Rev Saude Publica. 1999;33(2):198-205.

16. Eiser $C$, Morse R. The measurement of quality of life in children: past and future perspectives. J Dev Behav Pediatr. 2001;22(4):248-56.

17. Ingersoll GM, Marrero DG. A modified quality of life measure for youths: psychometric properties. Diabetes Educ. 1991;17(2):114-8.

18. Schau C, Stevens J, Dauphinee TL, Vecchio AD. The development and validation of the survey o attitudes toward statistics. Educ Psychol Meas. 1995;55(5):868-75

19. Sykes $V$. Validity and reliability qualitative research: a review of literature. J Mark Res Soc. 1990;32(3):24-36.

20. Pereira JCR. Escalas de medidas qualitativas. In: Pereira JCR. Análise de dados qualitativos: estratégias metodológicas para as ciências da saúde, humanas e sociais. $3^{\underline{a}}$ ed. São Paulo: EDUSP; 2004. p. 55-74.

21. Waller RA. Statistics: an introduction to numerical reasoning. San Francisco: Holden-Day Inc.; 1979.

22. Huang GH, Palta M, Allen C, LeCaire T, D'Alessio D. Self-rated healt among young people with type 1 diabetes in relation to risk factors in a longitudinal study. Am J Epidemiol. 2004;159(4):364-72.

23. O'Neil KJ, Jonnalagadda SS, Hopkins BL, Kicklighter JR. Quality of life and diabetes knowledge of young persons with type 1 diabetes: influence of treatment modalities and demographics. J Am Diet Assoc. 2005;105:85-91.
24. Maia FFR, Araújo LR. Aspectos psicológicos e controle glicêmico de um grupo de pacientes com diabetes mellitus tipo 1 em Minas Gerais. Arq Bras Endocrinol Metab. 2004;48(2):261-6

25. The Hvidore Study Group on Childhood Diabetes. Good metabolic control is associated with better quality of life in 2.101 adolescents with type 1 diabetes. Diabetes Care. 2001;24(11):1923-8.

26. Gray M, Boland EA, Yu C, Sullivan-Bolyai S, Tamborlane WV. Personal and family factors associated with quality of life in adolescents with diabetes. Diabetes Care. 1998;21(6):909-14

27. Faulkner MS. Quality of life for adolescents with type 1 diabetes: parental and youth perspectives. Pediatr Nurs. 2003;29(5):362-7.

28. Grupo Pediátrico ALAD. Diabetes mellitus: problemas en el diagnóstico y tratamiento em ninõs y adolescentes en Latinoamerica. Rev Assoc Latinoam Diabetes. 1999;(Suppl 1):136-41.

29. Moraes CM, Portella RB, Pinheiro VS, Oliveira MMS, Fuks AG, Cunha EF, Gomes MB. Prevalência de sobrepeso e obesidade em pacientes com diabetes tipo 1. Arq Bras Endocrinol Metab. 2003;47(6):677-83

30. Arcanjo CL, Piccirillo LJ, Machado IV, Andrade Jr CRM, Clemente EL, Gomes MB. Avaliação de dislipidemia e de índices antropométricos em pacientes com diabetes mellitus tipo 1. Arq Bras Endocrinol Metab. 2005;49(6):951-8.

31. Almeida HGG, Campos JJB, Kfouri C, Tanita MT, Dias AE, Souza MM. Perfil de pacientes diabéticos tipo 1: insulinoterapia e automonitorização. Rev Assoc Med Bras. 2002;48(2): 151-5.

32. Silveira VMF, Menezes AMB, Post CLA, Machado EC. Uma amostra de pacientes com diabetes tipo 1 no Sul do Brasil. Arq Bras Endocrinol Metab. 2001;45(5):433-40.

\section{Endereço para correspondência:}

Angelo Fontes Araújo

Rua Renato Mendonça, 175, apto. 202 - Edifício Maria

Helena, Brotas - 40285-440 - Salvador BA

E-mail: angelofamed@hotmail.com 